\title{
(Inter)religiöse Erziehung in der Migrationsgesellschaft
}

\author{
Thomas Eppenstein
}

As an impact of migration processes, we can realise a pluralism of religious organisations and movements. That is the reason for developing a basic attitude towards interreligious discourses in the following article, which includes a critical survey on experiences of adults in migration countries where one might identify cultural and religious pluralism. In addition and in contrast to these experiences, the everyday life of kids and youngsters will be analysed and reflected. The following aspects will also deal with the relation between religious challenges and an educational autonomy.

Since 2004 Thomas Eppenstein has taught Theories of Social Work and Educational Science at the Protestant University of Applied Sciences Rhineland-WestphaliaLippe in Bochum. Graduation under Micha Brumlik on the topic "Interkulturelle Kompetenz als pädagogische Kompetenz in der Migrationsgesellschaft", former teaching assignments at the Johann-Wolfgang-Goethe-University Frankfurt am Main, at the University of Heidelberg, the FH Wiesbaden, and long-term vocational occupations in contexts of migration and social work. His main research topics are intercultural education, religion and social work, pedagogy in migrational contexts (Migrationspädagogik) and global learning and change knowledge (Veränderungswissen).

\section{Einleitung}

Migrationen konfrontieren Zuwandernde und Ansässige mit der Erkenntnis, dass die jeweils eigenen, bislang als universell gültig erscheinenden religiösen oder weltanschaulichen Kernauffassungen, Zugehörigkeiten, kulturelle Praktiken und Wertvorstellungen im Ensemble pluralistischer Vielfalt lediglich als partikulare Möglichkeit mit womöglich eingeschränktem Gültigkeitswert von Belang sind. Gegenwärtige Migrationen sind damit Ausdruck und Folge der Pluralisierung der Moderne und ihren spezifischen weltgesellschaftlichen Dynamiken.

Die Erfahrung, dass die jeweils eigene bzw. gruppenbezogene religiöse oder weltanschauliche Kernauffassung in jeweiliger kultureller Einbettung und territorialer Verortung nur noch als jeweils eine partikulare Orientierung unter Vielen erlebt wird, lässt Bewältigungsstrategien und Bildungsprozesse unterschiedlicher Art entstehen. Entsprechende Verlusterfahrungen eigener vermeintlicher Monopolstellungen in der Moderne können zu Bescheidenheit, Dialogfähigkeit und zu einer Bildung führen, die die Menschenrechte vertritt und verteidigt. Im Rücken des empfundenen vermeintlichen Bedeutungsverlustes gedeihen jedoch auch Kulturalismus, Identitätspolitiken oder auch - zum Teil fundamentalistische 
- religiöse Suchbewegungen, die Perspektiven einnehmen, die politisch reaktionär, psychologisch regressiv und strukturell totalitär sind. So ist unter dem Signum des Religiösen zu unterscheiden zwischen einer Revitalisierung religiöser Orientierungen einerseits und einer politischen Instrumentalisierung von Religionen im militanten fundamentalistischen Extremismus andererseits.

Pädagogische Praxis und ihre erziehungswissenschaftliche Reflexion stehen in Kontexten migrationsbedingter Pluralisierung "migrationspädagogisch“ vor einer Reihe von Herausforderungen, entsprechende Veränderungen in Bildungskontexten und Erziehungskonzepten angemessen zu berücksichtigen, deskriptiv angemessen zu erfassen und normativ begründet zu orientieren. Aus einer religionspädagogischen Perspektive stellen sich ähnliche Fragen, wie nämlich in Hinblick auf Prozesse der Individualisierung und Pluralisierung von „Religion“ die je eigene religiöse Verortung situiert werden kann. So plädiert etwa Schweitzer aus evangelischer Sicht zuallererst für die Wahrnehmung einer sowohl weltanschaulischen wie religiösen Pluralisierung ${ }^{1}$ und argumentiert für die Perspektive einer religiösen Bildung, die ,die Möglichkeit einer dialogischen, von Toleranz bestimmten Begegnung“ unterstützt, denn: „Dialogische Verhältnisse zwischen verschiedenen Religionen und Weltanschauungen, die einander nun mehr und mehr im selben Land begegnen, sind ohne eine entsprechende Sprachund Urteilsfähigkeit kaum zu erreichen.“”2

In Hinblick auf die religiöse Vielfalt moderner Migrationsgesellschaften wird gelegentlich ein Wissensverlust bzw. Wissensdefizit sowohl in religionskundlicher Hinsicht, wie zum Stellenwert religiöser Orientierung für Individuen und soziale Gemeinschaften beklagt. Ob solches Nicht-Wissen tatsächlich um sich gegriffen hat, ob es vormals besser bestellt war, oder ob die Klage nicht eher einer allgemeinen Irritation über den diskursiven Bedeutungszuwachs von „Religiosität“ in säkularen Gesellschaften geschuldet ist, kann an dieser Stelle nicht eindeutig beantwortet werden. Gleichwohl trifft es zu, dass Konzepte differenzsensibler, interkultureller oder auch inklusiver Pädagogik bislang eher nachrangig Fragen religiöser Verortung aufgegriffen haben. Die interkulturelle Pädagogik habe bei allen Verdiensten, so Karin Amos, Probleme: „Als Programm, das in säkularen Gesellschaften entwickelt wurde, nimmt sie ,Religion' in einer sehr spezifischen Weise in den Blick. Religiöse Bildung ist in dieser Tradition kein Teil interkultureller Bildung. “3 Vice versa gilt für den didaktischen Bereich, dass in Hinblick auf den Gegenstand „Interkulturelle Kompetenz“ respektive „Interreligiöse Kompetenz" der Dialog zwischen Religionsdidaktik und interkultureller Didaktik noch eher spärlich ausgeprägt scheint. ${ }^{4}$

\footnotetext{
1 Vgl. Schweitzer 2016, S. 135.

2 Ebd., S. 172.

3 Amos 2015, S. 169.

4 Vgl. Schambeck 2013. Die Autorin greift zwar in ihrem „Basiswissen für Studium, Ausbildung und Beruf“" auf einschlägige Bezugsautorinnen zurück (Gogolin), bleibt aber
} 
Wenn hier von migrationsgesellschaftlichen Kontexten gesprochen wird, werden damit nicht spezifische Fokussierungen auf jeweilige „Ausländer“ in Nationalstaaten vorgenommen, sondern es werden damit Umstände zusammenfassend beschrieben, die sich durch einen hohen Grad der Mobilität, grenzüberschreitende Migrationen, diverse Integrations- und Desintegrationsprozesse im Zusammenhang von Globalisierung und weltweiten Interdependenzen innerhalb der Moderne auszeichnen, und die damit generelle Umstände bezeichnen. ${ }^{5}$

Die neuere Migrationssoziologie ${ }^{6}$ macht inzwischen deutlich, dass ergänzend zu Migrationsformen alten Typs, bei denen es um Aus- und Einwanderungsprozesse mit einer klaren Übereinstimmung zwischen territorialer Verortung und Sozialraum ging, transnationale soziale Bindungen quer zu unterschiedlichen Ländergrenzen transferiert werden, gestützt durch rasche Verkehrsanbindungen wie digitale Informations- und Kommunikationsmedien.

Eine deskriptive Analyse dessen, was diese Prozesse für Religionen und ihre institutionalisierten Manifestationen bewirken und wie dies in eine veränderte religiöse Bildung und Erziehung einfließt, kann empirische Tatbestände freilegen. Dazu gehören Phänomene transnationaler sozialer Netze, was transnationale Formen religiöser Bindung und Praxis einschließt.

Migration war und ist bis heute eine zentrale Ursache nicht allein für Kulturbegegnungen, sondern auch für die räumliche Nachbarschaft unterschiedlichster Religionsgemeinschaften, die sich oft in einer unvertrauten Diaspora-Situation wiederfinden. Zum anderen finden Begegnungen nicht allein mit anderen religiös verorteten Personen, Gruppen oder Institutionen statt, sondern die Konfrontationen in modernen Gesellschaften schließen die unterschiedlichen Ebenen nicht-religiösen oder auch areligiösen Charakters ein. ${ }^{7}$

Migration als Tatsache und konstitutiver Teil gesellschaftlicher Wirklichkeit ist von Diskursphänomenen zu unterscheiden, die Migration und Religion in ihren Zusammenhängen nicht analytisch freizulegen suchen, sondern eher in strategischer Absicht thematisieren.

In diskurskritischer Perspektive fällt ein dominanter werdendes Differenzkriterium der religiösen Zugehörigkeit ins Auge, vor allem hinsichtlich eines neu aufscheinenden Bedrohungsszenarios, das mit Muslimen bzw. einzelnen Strö-

einem begegnungspädagogischen Ansatz verhaftet. Die aus katholischer Perspektive stark normativ ausgerichtete Perspektive, Eigenes und Fremdes in einem theologischen Differenzmodell im Medium der Liebe Gottes zu thematisieren, wird nicht an die vorgängigen differenztheoretischen und differenzkritischen Positionen der Allgemeinen Erziehungswissenschaft, etwa bei Lutz und Wenning angeschlossen.

5 Vgl. Eppenstein 2003, S. 117-125.

6 Vgl. Pries 2001 und 2006.

7 Vgl. zu den normativen Implikationen Schweitzer 2016, S. 178 ff. 
mungen im Islam assoziiert wird. ${ }^{8}$ „Religion“ avanciert so einmal zu einem Differenzkriterium erster Güte, droht andererseits im Chor normativer Vielfaltspostulate als beliebiges Differenzmerkmal unter vielen verkannt zu werden. Differenzmuster zwischen religiös gebundenen und sich dagegen absetzenden Positionen werden zum Kennzeichen sozialer Wirklichkeit(en).

Die erziehungswissenschaftlichen Reaktionen auf die Migrationstatsachen des letzten halben Jahrhunderts folgten zunächst einem kompensatorischen ,ausländerpädagogischen“ Ansatz, der sich - horizontal - auf Differenzen der sozialen Lage konzentrierte, gefolgt von der Perspektive einer ,interkulturellen Pädagogik“, die nun - vertikal - auf Lernprozesse von Zugewanderten wie Einheimischen gleichermaßen in Hinblick auf kulturelle Differenzen setzte. Eine Reflexion kulturalistischer Engführungen führte zu einer reflexiven Interkulturalität bzw. nun als „Migrationspädagogik“ bezeichneten Orientierung, die Migrationserfahrungen einbezog und auch rassismuskritische Implikationen aufgriff. ${ }^{9}$ Die interkulturelle Pädagogik nahm lange Zeit die Fragen um religiöse Orientierungen von Kindern, Heranwachsenden und Erwachsenen eher beiläufig in Kontexten kultureller Diversität und einem allgemeinen Postulat auf, wonach es gelte, Vielfalt anzuerkennen. Dabei wurden Spezifika religiöser Bildung jedoch kaum hinreichend gewürdigt und auch die Verschiebungen in öffentlichen Diskursen um den Bedeutungszuwachs des „Religiösen“ konnten eher nur reaktiv erfasst werden.

So changieren pädagogische Praktiken und erziehungswissenschaftliche Reflexionen häufig zwischen dem Versprechen, migrationsgesellschaftliche Bewältigungsaufgaben generell lösen zu helfen und der Überforderung, dies im je spezifischen Fall und Feld tatsächlich zu leisten. ${ }^{10}$,Jetzt auch noch interreligiöse Erziehung?" werden sich praktizierende Pädagoginnen und Erzieher möglicherweise fragen, denn die Hochschätzung relevanter Bildungsräume hat eine Fülle an Erwartungen angehäuft: So gilt etwa Kindheit als Terrain für Bildungsinvestitionen, die Kinder frühzeitig als künftige Problemlöser stark machen sollen. Sprachliche, musische und naturwissenschaftliche Kompetenzen sollen nicht brach liegen und der Explorationstrieb soll sich in anregender Umgebung entwickeln können. Gleichzeitig sind professionelle Pädagogen und Erzieherinnen mit der Pluralität von lebensweltlichen Kontexten ihres Stadtteils, der Familien und Elternschaften konfrontiert, die zum Teil unterschiedliche Erwartungen einbringen. Allein z. B. die Frage, was und wie Kinder während ihres Aufenthalts in Kita, Schule oder außerschulischer Einrichtung essen sollen oder dürfen, stellt in Zeiten divergierender Lebenskonzepte, pluraler weltanschaulicher oder religiöser Vorschriften, verbreiteter Allergien usw. eine Herausforderung dar. Sie

$8 \quad$ Vgl. Bahners 2011 und Bade 2013.

9 Vgl. Eppenstein 2003, S. 117 und 177; Eppenstein / Kiesel 2004, S. 47-49 oder Mecheril et al. 2010, S. 11.

10 Vgl. Eppenstein 2007/2013, S. 29. 
fordert Kompetenzen unter Berücksichtigung von Kenntnissen über gesunde Ernährung oder pädagogisch vertretbaren alternativen Konzepten, entsprechenden Regelungen mit den beteiligten Akteuren, z.B. Eltern, Geldgebern, Kindern etc. auch jeweils aushandeln zu können. Dasselbe gilt für Schulen und die vielfältigen Formen außerschulischer Bildung und sozialpädagogischer Konzepte, die sich auf eine pluralisierte Schülerschaft inklusiv zu beziehen haben.

Wenn hier in Grundlagen zu Fragen interreligiöser Erziehung eingeführt wird, so soll dies einen solchen denkbaren „Überforderungskatalog“ nicht noch weiter anfüllen, sondern will Reflexionen, praxisrelevante theoretische Elemente, Begründungen und Perspektiven bereit stellen, die es erleichtern, im Zusammenhang mit der gegebenen religiösen Pluralisierung eine entsprechende pädagogische Praxis auszurichten und zu begründen.

Die Frage nach einer definitorischen Bestimmung des „Interreligiösen“ zieht dabei Fragen nach Interdependenzen verschiedener Ebenen nach sich: $\mathrm{Zu}$ unterscheiden sind die öffentliche und private Sphäre, die Ebene der Struktur (säkulare, staatlich oder innerhalb der jeweiligen religiösen Gemeinde/Gemeinschaft), sowie der Ebene der Rechte (Verfassung) sowohl auf staatlicher wie auf religiöser Seite. Wer ist da jeweils in welchem Dialog Sprecher/-in von was? Dies gilt es - anstelle einer lexikalischen Definition - jeweils zu rekonstruieren bzw. offen zu legen.

Während politische Positionen in einer demokratischen Gesellschaft changieren können oder gar sollen (Wechselwähler), werden religiöse Haltungen als mit der subjektiven Identität verwoben und als gerade nicht austauschbar erachtet. Sie sind oft die verbleibende dominante Orientierung, an der Zugehörigkeit, Tradition und Kontinuität von Angehörigen einer Gesellschaft gemessen wird, die ansonsten auf die Mobilität, Individualität und Wandlungsfähigkeit ihrer Mitglieder stolz ist und diese explizit fördert. Insofern kommt der Gestalt des „Interreligiösen“ nicht allein eine theologische Dimension zu, sondern ebenso eine soziale und kommunikative Aufgabe.

Vor diesem Hintergrund werden in diesem Beitrag zunächst Begründungszusammenhänge für eine (inter)religiöse Erziehung beschrieben (1). Es folgt eine Reflexion der Erfahrungen, wie sie zunächst allgemein in Einwanderungsgesellschaften mit ihrer kulturellen und religiösen Pluralisierung Erwachsene machen, um davon ausgehend und zum Teil damit kontrastierend die spezifischen Erfahrungsdimensionen von Kindern und Heranwachsenden herauszuarbeiten (2). Die folgenden Abschnitte behandeln die spezifischen pädagogischen Implikationen und diskutieren abschließend das Verhältnis von „religiöser Zumutung“ und einer „Erziehung zur Mündigkeit“ (3). 


\section{2. (Inter)Religiöse Erziehung - Begründungszusammenhänge für eine unklare pädagogische Perspektive}

Erziehungswissenschaftliche Forschungsfragen ergeben sich aus praktischen Handlungszusammenhängen wie diesem:

In einer Weiterbildung zur interkulturellen Kompetenz für Lehrkräfte und Erzieherinnen (2014 in Frankfurt am Main), die selbst einen sogenannten „Migrationshintergrund" biografisch vorzuweisen haben, stellt ein junger Lehrer folgende Begebenheit zur Diskussion:

In meiner neuen Klasse fragt mich ein 10jähriger Schüler - ich glaub' seine Eltern war'n aus Marokko - ob ich Muslim sei. Ich wusste erst nicht (Pause), und sagte dann: Ja, bin ich. Da strahlt der und sagt „guuut“. Ich wusste nicht (Pause), ich hab' ihm gesagt, ich bin doch Mathematik- und Sportlehrer. ${ }^{11}$

Die Erzählung erfolgte im Kontext von Beispielen für praktische Problemstellungen und wurde von den teilnehmenden Kollegen und Kolleginnen mit Gesten und Bekundungen aufgenommen, die signalisierten, man wisse nur zu gut, was hier das Problem sei. Eine vertiefende Erörterung oder Nachfragen ergaben sich jedoch nicht.

Textstelle und diskursiver Kontext lassen nun mehrere Interpretationen $\mathrm{zu}$ :

Die Nachfrage des Schülers irritiert die Lehrkraft. Deutlich wird diese Irritation an der Passage ,ich wusste erst nicht (...)“. Die Gründe für die Irritation bleiben jedoch offen. Als Problemstellung könnte gelten,

- dass die Lehrkraft einen Rollenkonflikt fürchtet. Sein Selbstverständnis in der Lehrerrolle verbietet ihm, Identitätsaspekte außerhalb seiner Fachlehrerschaft dem Schüler mitzuteilen.

- dass der Lehrer ein ambivalentes oder gar gestörtes Verhältnis zu seiner eigenen religiösen Verortung hat oder dass er gelernt hat, diese vor anderen zurückhaltend zu äußern.

- dass der Schüler Nähe und Akzeptanz zu seinem neuen Lehrer sucht und sich dieser über religiöse Zugehörigkeit zu vergewissern sucht.

- dass der Schüler die Trennung von Staat und Religion nicht kennt, nicht kapiert, nicht akzeptiert... und damit Hilflosigkeit bei seinem Lehrer auslöst, der selber bemüht ist, die Bereiche sauber zu trennen.

- dass der Lehrer Auskunft geben wollte, obwohl es nicht zu seinem Unterrichtsfach gehört, und nun eine Art „Kumpanei“ seitens des Schülers fürchtet, an der ihm gar nicht gelegen ist.

- dass der Schüler fehlenden Islamkundeunterricht durch seine Annäherungsversuche an den Sport- und Mathelehrer als „ethnisch“ in Frage kommenden Akteur zu kompensieren sucht.

11 Transkription unmittelbar im Anschluss an die Aussage. 
- Dass dem Lehrer situativ klar wird, dass im Moment dieses „Bekenntnisses“ die Frage aufgeworfen wird, wie andere Schülerinnen und Schüler anderer religiöser Zugehörigkeit oder gar keiner religiösen Verortung darauf reagieren werden.

- Dass dem Schüler an einer Art Koalitionen-Bildung gelegen ist oder aber dies gar nicht der Fall ist. Dem Lehrer bleibt daher das Motiv für die Frage unklar.

$-\ldots$

Das hermeneutische Sampling kann hier unterbrochen werden, denn das Beispiel soll lediglich illustrieren, dass pädagogische Interaktionen im Normalfall ungeklärte interpretative Bedeutungszusammenhänge aufnehmen und diese in einen Sinnhorizont von Lern- und Bildungsperspektiven transferieren wollen, mithin kontrafaktisch Determination vorgeben, wo Kontingenz die Wirkungsmuster ausmacht.

\subsection{Religiosität: Pluralität von Bedeutungszuschreibungen}

Im vorliegenden Beispielfall kann der - situativ etwas hilflose - Abbruch der Kommunikation in der Lehrer-Schüler-Interaktion als Problemstellung zweiten Grades festgemacht werden. Dies meint, dass nicht die Äußerungen des Schülers als Problem in den Blick genommen werden, sondern die Irritation und deren mögliche Gründe, die sie bei der Lehrkraft ausgelöst hat. Hier kann die pädagogische Selbstverständigung anhand von Wissen um Religion und Religiosität helfen, die über eine einfache Polarität hinausgeht: Entweder man ist religiös und Anhänger oder Mitglied einer bestimmten Religionsgemeinschaft, deren Essentials man bei Bedarf vertritt oder weitergibt, oder man ist es nicht und verweist alles, was mit Religion zu tun hat in die Sphäre des Privaten, der Opiate oder im säkularen Fundamentalismus in den Bereich des Ursprungs aller Übel. Wer Religion nur feindlich gegenüber steht, wird sie auch als soziales Phänomen nicht verstehen. Auch Anhänger einer rationalistischen Weltsicht wie Albert Einstein, der in Anlehnung an Spinoza einem unpersönlichen Gottesbegriff folgte, konzedierte 1929:

Ein anderes ist die Frage, ob der Glaube an einen persönlichen Gott bekämpft werden soll. [...] Ich selbst würde mich an so etwas nicht beteiligen. Denn ein solcher Glaube erscheint mir immer noch besser als überhaupt keine transzendentale Lebensauffassung, und ich frage mich, ob es je gelingen würde, der Mehrheit der Menschen ein vortrefflicheres Mittel zur Verfügung zu stellen, um ihre metaphysischen Bedürfnisse zu befriedigen. $^{12}$

Jammer 1995, S. 34. 
Pädagogische Praxis als personenorientierte, intentionale Handlungsform ist auf Praxisforschung verwiesen, die im Feld der religiösen Erziehung wie außerhalb dieses Feldes mit „Religion“ als intervenierender Variable oder als Differenzkategorie darauf angewiesen ist, unterschiedliche Bedeutungszuschreibungen des Religiösen zu kennen, zu unterscheiden und sich reflexiv damit im Verhältnis zur jeweils eigenen Verortung auseinander zu setzen.

Hierzu folgende Aspekte, die für eine kategoriale Einordnung bedeutsam sein können:

- Zur vermeintlichen Konkurrenz wissenschaftlicher Wahrheitsansprüche mit religiösen Überzeugungen gilt es, das Missverständnis aufzuklären gegenüber religiösen Aussagen und Bekenntnissen, die nicht im Modus wissenschaftlicher Theorien oder empirischer Daten zu verstehen sind, sondern als Artikulationen fundamentaler Lebensorientierungen. Religiöse Gewissheiten folgen einer spezifischen Rationalität, die der Logik von Verifikation und Falsifikation nicht folgt und können demnach weniger als naturwissenschaftliche Wahrheiten, denn als Ausdruck interpersonaler Vertrauensverhältnisse gedeutet werden. ${ }^{13}$

- „Religion“ kann ferner als Sinnsystem gelten, das das Leben religiöser Menschen zu strukturieren vermag. „In der Religion geht es um eine existenzielle Rückbindung an eine andere - nicht empirische - letzte Wirklichkeit" die zwar außerhalb der Zugriffsmöglichkeiten von Menschen liege, aber dennoch in dessen Lebenswirklichkeit Wirkungen zeigt. ${ }^{14}$

- Hans Joas erkennt treffend als ein Spezifikum der Kommunikation über Werte, wie sie ja grundlegend und tief im religiösen Denken und Handeln angelegt sind: „Über Werte zu sprechen, setzt die Berücksichtigung der affektiven Intensität unserer Bindung an sie voraus. "15 Mit der Bedeutung solcher Bindungen rühren Werte an Kernüberzeugungen, die über bloße Meinung oder rationale Argumente hinausgehen: „Unsere Erwartung an den Zuhörer ist in diesem Fall eine ganz andere als die, von der die Diskurstheorie spricht. Es ist weder unsere Erwartung noch gar unsere Intention, daß der Zuhörer von uns in dem Sinne überzeugt wird, daß er unsere Gefühle teilt [...]. " ${ }^{16}$ Das bescheidene Ziel wäre also Plausibilität und nicht Konsens: „Weil Werte ohne Bindung nur Behauptungen sind, Werte mit Bindung aber nicht wie bloße Behauptungen behandelt werden können, ist für die Logik einer Kommunikation über sie die Berücksichtigung der Konstitution unserer Bindung an sie das erste wesentliche Merkmal.“17

13 Vgl. Rentsch 2008.

14 Vgl. Bohmeyer 2009, S. 442.

15 Joas 2011, S. 256.

16 Ebd., S. 257.

17 Ebd. 
- Aus wissenssoziologischer Perspektive lässt sich mit Luckmann Religion allgemein als etwas betrachten, das mit der menschlichen Fähigkeit zum Transzendieren verwoben ist ${ }^{18}$, und theoretisch kann "Transzendenzerfahrung“ an die phänomenologische Strukturanalyse von Lebenswelt und die unterscheidbaren Bereiche von Wirklichkeit bei Alfred Schütz angeschlossen werden. ${ }^{19}$

Pädagogische Praxis ist an Erfahrungshorizonte der Educandes verwiesen und sollte das, was Einstein durchaus nüchtern als „transzendentale Lebensauffassung" beschreibt, auch bei Berücksichtigung religiöser oder weltanschaulicher Neutralitätsgebote nicht übergehen. Zum phänomenologischen Konzept der „Erfahrungstranszendenzen“ erläutert Knoblauch, der Begriff beziehe „sich auf diejenigen Aspekte menschlichen Erfahrens, die das übersteigen, was sich in der Unmittelbarkeit des Erfahrens selbst präsentiert " ${ }^{\text {"20 }}$. Aus religionssoziologischer Perspektive resümiert er in einem Beitrag zur "Soziologie der Spiritualität“ Quellen ,alternativer Religiosität [...], die sich aus den nicht hegemonialen (östlichen, archaischen, okkulten, mystischen etc.) Traditionen der Religion zusammensetzen“ und die durch eine „Distanz zur Dogmatik religiöser Großorganisationen" ausgezeichnet seien. ${ }^{21}$ Das hier beanspruchte Individualisierungstheorem analysiert Phänomene neuer ,alternativer" religiöser Verortungen derart, dass einerseits vollzogene Individualisierungsschübe, andererseits eine religionsaffine Bedürfnisstruktur als ursächlich gelten.

\subsection{Erziehungswissenschaftliche Vergewisserungen}

Eine erziehungswissenschaftliche Annäherung an Fragestellungen zu „Religion und Migration“ ist auf unterschiedlichen Ebenen möglich.

Deskriptiv können zunächst Phänomene beschrieben und analytisch befragt werden, die um Migrationsfolgen, der damit einhergehenden Pluralisierung von Lebenswelten und migrationsgesellschaftliche Bewältigungsaufgaben generell kreisen und die sich zum anderen mit der Pluralisierung religiöser und weltanschaulicher Kernüberzeugungen kreuzen, die im Vollzug von Modernisierung und Säkularisierung eintritt. Solange Kontexte einer dominanten Kultur, Religion, regionaler Gebundenheit und zeitlicher Überschaubarkeit beieinander lagen und eine Art lebensweltliche Einheit ergaben - was auch in der zurückliegenden Geschichte selten auf Dauer der Fall war - schienen Fragen der Erziehung insofern undramatisch, als es wie selbstverständlich um Absichten ging,

18 Vgl. Luckmann 1991.

19 Vgl. Schütz 2003.

20 Knoblauch 2005, S. 125.

21 Ebd., S. 123. 
die nachwachsende Generation in diese bestehenden Selbstverständlichkeiten einzupassen und für deren Fortführung oder weiteren Gestaltung auszustatten. Erziehung konnte nur solange als Assimilation der nachwachsenden Generation an die bestehende gelten, als letzterer ein Wissen zugeschrieben werden konnte, das dem der Jüngeren voraus war und das beanspruchen durfte, einigermaßen verlässliche Orientierungen für Gegenwart und Zukunft zu gewährleisten. Dies gilt auch in der aufkommenden Moderne vor der sogenannten Globalisierung, die bereits mit Comenius, später etwa mit Rousseau und Kant „Erziehung“ als Bedingung für die noch ausstehende Vervollkommnung des Menschen und menschlicher Verhältnisse konzipiert. Mit der Beschleunigung von Veränderungsprozessen - auch im technologischen Bereich von zunächst Industrialisierung und heute Digitalisierung - verschieben sich die intergenerativen Bildungsverläufe auf radikale Weise. Während in vormodernen Gesellschaften und Produktionsweisen Bildung noch im Zusammenleben und Arbeiten durch meist unmittelbare „Präsentationen“ erfolgte, wurde sie im Vollzug der Trennungen von Kindheiten und Erwachsenenwelt durch „Repräsentationen“"22 anhand von didaktischen Materialien, Bildern und Texten ersetzt und braucht heute so etwas wie „Präpräsentationen“, also auf die Kontingenz einer sich rasch verändernden Zukunft gerichteten Ausrichtung. Erziehung und Bildung zielen in der fortgeschrittenen Moderne also auf Kompetenzen der Kontingenzbewältigung in Hinblick auf risikogesellschaftliche Verhältnisse trotz eines Zuwachses an technologischer Verfügbarkeit zentraler Lebensbereiche. Religion als eine kulturell lang tradierte Form von Kontingenzbewältigung tritt gewissermaßen mit einer säkular strukturierten Pädagogik in Konkurrenz, die Kontingenzbewältigung auf „Sichtflug“ in eine unüberschaubare Zukunft mittels Kompetenzerwerb zu erreichen sucht.

Religiöse Bildung, einmal als Wissensbestand, ein andermal als Identitätsmerkmal erfordert unter diesen Vorzeichen eine differenziertere Ausrichtung als unter Bedingungen, bei denen religiöse Verortung, Alltagskultur und Lebenswelt ineinander flossen: Man ist katholisch und lebt in einem katholischen Land, oder man ist evangelisch und wohnt in einer protestantischen Region, man ist Grieche und mehrheitlich orthodox. Die „muslimische Welt" galt lange als eine auch territorial fassbare Größe. Solange also Kultur, Religion und territoriale Einheit mehr oder weniger einheitlich erfahren werden, solange die „Fremden“ und „Anderen" nicht nur anders, sondern auch woanders sind, scheinen die Zumutungen einer pluralen Welt erträglich. Von diesem Schein lässt sich das rassistische Weltbild bis heute blenden, wenn es eine "heile Welt" voneinander getrennt existierender Rassen und Völker imaginiert, deren Vermischung die größte Sünde darstellt, weil sie die konstruierten Hierarchien und Differenzen zwischen ihnen zu verunreinigen drohen. Diesem Schein folgen auch alte und neue Fundamen-

22 Vgl. Mollenhauer 1983. 
talismen mit und ohne religiöse Vorzeichen, die mit Riesebrodt ${ }^{23}$ als Phänomen der Moderne und gegen diese Moderne antreten, um eine nach ihren Vorstellungen ideale Welt zu errichten. Religiöse Verortungen sind mit rassistischen Optionen nicht zu verwechseln oder gar gleichzusetzen. Für Religionsgemeinschaften entsteht jedoch im Wissen um die mögliche politische Instrumentalisierung religiöser Orientierungen eine besondere Verantwortung, Fragen friedlicher und dialogischer Koexistenz auch dann einzubeziehen, wenn ihre jeweilige Orthodoxie Alleinvertretungsansprüche postuliert bzw. wenn Grenzen und Unvereinbarkeiten interreligiöser Verständigung in der Sache begründet liegen. Damit geraten ,die Religionen' selbst in den Strudel der durch die Moderne in Gang gesetzten Veränderungen. Wenn nach ,interreligiöser“ Erziehung in Migrationsgesellschaften gefragt wird, stellt sich nicht nur die Frage nach einer veränderten religiösen Erziehung, sondern man muss sich klar machen, dass es eine unveränderte Beibehaltung religiöser Kernauffassungen in der postsäkularen Gesellschaft nur unter der Bedingung zu geben scheint, als es ihren Vertretern und Vertreterinnen gelingt, diese auch immer in die Sprache der Anderen, einschließlich religiös unmusikalischer Menschen zu übersetzen. Am deutlichsten scheinen diese Zusammenhänge, wenn man die jeweiligen Konflikte zwischen „orthodoxen“ und ,liberalen“ Strömungen betrachtet: An einem Witz zum Reformjudentum der ersten Hälfte des 20. Jahrhunderts wird das lebensweltliche Dilemma deutlich:

Der kleine Moische, sechs Jahre alt, kommt weinend aus dem Religionsunterricht nach Haus. Warum er weine, fragen ihn die hoch assimilierten Eltern, die das Judentum kaum mehr praktizieren. „Der Rabbi will, dass wir Kinder in einem jüdischen Zuhause aufwachsen. Aber ich will doch bei euch bleiben! ${ }^{24}$

Die Pointe liegt hier in der Uneindeutigkeit der Auslegung: Hat der Junge den Rabbi missverstanden, oder hat er ihn nur zu gut verstanden? Geht es um die Bestätigung des Bundes im Sinne des Judentums quer zu allen Facetten und pluralen Ausformungen jüdischen Lebens in der Moderne oder geht es um eine alternative Entscheidung zwischen ,wahrer Religion“ und familiärer Lebenssituation, in der das „Jüdisch-Sein“ in unterscheidbaren Varianten gelebt werden kann?

„Religion“ und ihre Vermittlung durch Erziehung und Sozialisation kann entsprechend in unterschiedlichen Hinsichten betrachtet werden. Sie hat viele Gesichter und Dimensionen:

- Eine lebensweltliche Dimension, die sich im familiären Alltag vollzieht und jeweilige Auslegungen, Praktiken, Varianten mitvollzieht.

23 Vgl. Riesebrodt 2000.
24 Joffe 2015, S. 120 f. 
- Eine Wissensdimension um religiöse Narrative und die zugrundeliegenden Texte.

- Eine Dimension der persönlichen Transzendenzerfahrung, Glaubensdimension oder auch spiritueller Elemente.

- Eine soziale Dimension in der Verwobenheit mit politischen und sozialen Fragen.

\subsection{Pädagogische Fragen}

Die Erziehungswirklichkeit in postsäkularen Migrationsgesellschaften ist in Hinblick auf eine zweite Ebene jedoch auch danach zu befragen, welche normativen Perspektiven hier eine Rolle spielen. Was soll in interreligiösen Bildungsprozessen gebildet werden? Wer soll und kann hier was von wem lernen und welche ethischen Grundlagen sind relevant, wenn doch religiöse Vorschriften selbst jeweils einen Haushalt entsprechender Gesetze und Vorschriften vorhalten?

Heiner Bielefeld weist zu Recht darauf hin, dass der religiöse Pluralismus ,sehr viel weiter geht als die im öffentlichen Bewusstsein immer hervorgehobenen Differenzen zwischen Muslimen und Christen“, und dass es „nicht nur einen Pluralismus der Religionen gibt, sondern auch einen Pluralismus der Umgangsformen mit Religion“"25. Damit sind Agnostiker, areligiös oder antireligiös eingestellte Menschen in die Fragestellung inkludiert, wie mit konfliktgenerierenden Aspekten dieser Pluralität zu verfahren ist. In normativer Hinsicht impliziert dies Lernziele im Rahmen eines Bildungskonzeptes, das mit der religiösen Bildung eine ,interreligiöse“ Perspektive unter den Vorzeichen des Kanons der Menschenrechte einbezieht.

Für interkulturelle Konzepte stellt sich die Frage nach dem Umgang mit Differenzen in der postsäkularen Gesellschaft, die dadurch gekennzeichnet ist, dass „sie sich auf das Fortbestehen religiöser Gemeinschaften in einer sich fortwährend säkularisierenden Umgebung “26 einzustellen hat. Die interkulturelle Perspektive lässt sich erweitern auf die von Habermas eingeforderten Prozesse gegenseitigen Lernens zwischen säkularisierten und religiös grundierten Bürgern. Denn die „religiöse Signatur der Moderne“ ${ }^{\text {27 }}$ zeichnet sich nicht durch ein Verschwinden der Religion aus, sondern das Religiöse wird zunehmend von seinen Bezügen der Vergemeinschaftung entkoppelt und verlagert sich von den Institutionen in das Subjekt hinein. ${ }^{28}$ Gleichwohl behalten religiöse Institutionen gerade dort ihre Bedeutung, wo sie für den Erhalt eines ,kulturell geteilten Be-

25 Bielefeld 2007, S. 22.

26 Habermas 2001, S. 13.

27 Bohmeyer 2009, S. 445.

28 Vgl. Kaufmann 2008. 
standes an religiöser Praxis“ sorgen, der sich vor allem im Vollzug und bei der Bewältigung biografischer Übergänge im Lebenslauf als erforderlich erweist. ${ }^{29}$

Erschwerend dabei ist, dass eine Klärung darüber, was in einem originären Sinne ,interreligiös“ ist, - sein kann oder sein soll, kaum möglich erscheint. Wohl aber lassen sich verschiedene Praktiken und Konzepte beschreiben und unterscheiden, die sich selbst als ,interreligiös“ einstufen, z. B. der ,interreligiöse Dialog“ zwischen erwachsenen Vertretern von Religionsgemeinschaften, oder ganz unterschiedliche praktische Versuche, auf die religiöse Pluralisierung jeweils pädagogisch zu reagieren. Es soll hier nicht der Ort sein, dies im Einzelnen zu rekonstruieren, aber grob lassen sich etwa für den elementarpädagogischen Bereich in der Bundesrepublik Deutschland mindestens drei Richtungen ausmachen:

- Eine Konzeption fußt auf der Aufrechterhaltung einer geklärten eigenen institutionellen Identität, z. B. als Kindergarten in einer bestimmten konfessionellen Trägerschaft, wobei religiöse Inhalte, Feste, Rituale und Feiertage etc. beibehalten, und Kinder (und Eltern) anderer Religionszugehörigkeit gegebenenfalls zur Teilnahme „eingeladen“ werden. Vorteile dieser Richtung liegen in ihrer Eindeutigkeit und Klarheit gegenüber allen Beteiligten, Nachteile in Erfahrungen des Ausgeschlossen-Seins der Kinder, sofern sie einer anderen Religion angehören.

- Eine weitere Richtung beansprucht ebenfalls Perspektiven religiöser Erziehung, will aber Kinder jeweils anderer Religionszugehörigkeiten durch veränderte und flexible Arrangements z. B. bei der Gestaltung von Feiern und Festen einbeziehen. Vorteile werden hier in einer diskriminierungsfreien, alle irgendwie einschließenden Praxis gesehen, Nachteile betreffen den Vorhalt des Synkretismus von der einen Seite oder eines illegitimen Vereinnahmungsversuchs von der anderen.

- Eine dritte Konzeption schließlich vermeidet religiöse und interreligiöse Anfragen oder feiert - etwa in einer überkonfessionellen städtischen Einrichtung diverse profane und religiöse Feste „quer Beet“ (Halloween, Ramadan, Weihnachten etc.). Der Vorteil liegt hier in einem Bemühen, die Vielfalt sichtbar werden zu lassen, allerdings um den Preis, dass die jeweils originäre religiöse Erziehung woanders - z.B. in der Familie - statt zu finden hat und in der Kita lediglich eine Art „Potpourri“ religiös-kultureller Anleihen erfolgen kann.

Elementarpädagogische Bildungsinstitutionen sollten sich also strukturell wie konzeptionell ausweisen können, denn sie bilden nicht von selbst einen Lebensund Erfahrungsraum für religiöse Erziehung bzw. interreligiöse Erziehung.

Im Bereich der Schulen ist auf die oft mühsam erscheinenden Entwicklungen in den einzelnen Bundesländern zu verweisen, einen islamischen Religionsun- 
terricht zu implementieren. Komplementär wäre die Einrichtung von Lehrstühlen an einzelnen Hochschulen zu nennen.

$\mathrm{Zu}$ den Grundlagen einer Begründung (inter)religiöser Erziehung gehört zunächst die Einsicht, dass religiöse Orientierungen maßgeblichen Anteil daran haben, wie Menschen ihre jeweils eigene Lebensführung und ihr Zusammenleben untereinander ausrichten. Religionen werden und wurden sozial bedeutsam, wenn sie Einfluss auf normative und ethische Vorstellungen und Praktiken haben. Religiöse Praktiken werden dabei von Alltagspraktiken unterschieden, wenngleich gerade lebensweltliche Vertrautheiten und Bewältigungsmuster im Alltag von Einflüssen religiöser Traditionsbildung durchdrungen sind. So kann man z. B. die ,Tugend“ der Pünktlichkeit in westlichen (post)industriellen Gesellschaften auf Wechselwirklungen mit der ,protestantischen Ethik“30 im Kontext mit der Entstehung der bürgerlichen Gesellschaft zurückführen. Dies gilt übrigens auch dann, wenn die Akteure sich selbst als ,nicht-religiös“, als ,,anti-religiös“, oder als „skeptisch“ einordnen. In Bildungs- und Erziehungskontexten als Alltagspraxis greifen Dimensionen von Religion und Religiosität auf die profanen Bereiche des Lebens über; hier geht es also nicht zuvorderst darum, wie einzelne Menschen ihre Fragen zu Transzendenz, geistiger Wirklichkeit, Gott usw. klären, sondern um die Beachtung relevanter Wechselwirkungen zwischen sakraler, liturgischer, kirchlicher usw. Ebene und den Realitäten von Alltag und Lebenswelt. Mit Blick auf Bildungsorte und Institutionen wäre hier zu unterscheiden zwischen etwa konfessionellen oder nicht-konfessionellen Kindergärten oder Schulen, sowie Orten der religiösen Unterweisung und dem jeweiligen Alltag von Heranwachsenden auch außerhalb solcher Institutionen.

Die Pluralität gelebter Religiosität in Bezug zur gelehrten Religion rekonstruierend historisch darzustellen, ist kaum zu bewältigen, wohl aber bietet die Pluralität der Lebenswelten Anlass, den jeweiligen Bedeutungshorizont von „Religion“ für die Beteiligten zu verstehen und, sofern er von den jeweils eigenen Gewissheiten abweicht, tolerieren zu lernen. Hierbei erweist sich bereits oft die kulturelle Pluralität innerhalb einer Religion als Herausforderung.

Ein zentraler Begründungszusammenhang für eine religiöse Erziehung, die auch die jeweils anderen Religionen anerkennend in den Blick nimmt, dialogisch ausgerichtet also als ,interreligiöse“ Erziehung bezeichnet werden kann, ergibt sich, wie eingangs erwähnt, aus der kulturellen und religiösen Pluralisierung, wie sie unter anderem durch Migrations- und Einwanderungsprozesse sichtbar geworden ist. In Modernisierungs- und Globalisierungsprozessen, die hierzu in engem Zusammenhang stehen, müssen Menschen ihre selbständige Lebensführung unter potenziell riskanten Bedingungen immer wieder neu wiedergewinnen und gleichzeitig damit zurechtkommen, dass die je eigenen kulturellen und religiösen Traditionen mit denen der anderen - jetzt auch im sozialen Nahraum kollidieren können. Die soziale Dimension religiöser Orientierungen kann sich

30 Vgl. Weber 1920/2004. 
dabei - unabhängig von der jeweiligen Religion selbst - in ganz unterschiedlicher Hinsicht und Perspektive manifestieren:

- als Bindung an eine Religionsgemeinschaft mit ihrer je eigenen Geschichte;

- als Resultat erfahrener Erziehung, erlebter Sozialisation oder vollzogener Bildungsprozesse;

- als Strukturierung der eigenen Lebenswelt und des Alltagsverständnisses;

- als sozial wirksame Hoffnung, Aufgabe oder Lebensorientierung;

- als kondensierte Form der Gebote Gottes (in den monotheistischen Religionen) in der jeweiligen Lebensform $;^{31}$

- als pseudoreligiöse Selbstrechtfertigungen im Fundamentalismus.

Schließlich kann eine „interreligiöse“ Dimension religiöser Erziehung mit den Spannungen und Entwicklungen säkularisierter Gesellschaften begründet werden. Der kanadische Philosoph Charles Taylor hat sich mit dem Verhältnis kultureller und religiöser Minderheiten zum kanadischen Staat beschäftigt und unterscheidet in seiner jüngsten Studie zum ,säkularen Zeitalter“32 drei Formen der „Säkularisation“ hinsichtlich der Bedeutung von Religionen:

1. Die Trennung und Gewaltenteilung von Staat und Kirche lasse die öffentliche Bedeutung der Religionen schwinden.

2. Der Prozess der Säkularisierung bedingte ein Schwinden religiöser Überzeugungen und Bindungen, die sich z. B. in der geringer werdenden Anzahl von Gottesdienstbesuchern zeige. Dies impliziere auch einen Wissensverlust über zentrale Bedeutungen etwa von Feiertagen und den dazu gehörigen Narrativen vormals dominierender Religionen, auch der eigenen.

3. Begünstigt durch den hohen Grad an Ausdifferenzierung in modernen Gesellschaften herrsche eine Vielzahl von Glaubensangeboten, die im Sinne der Religionsfreiheit und innerhalb rechtsstaatlicher Grenzen zwar allesamt erlaubt seien, sich aber gegenseitig entwerten.

Gleichsam konstatiert Taylor ein Fortleben der Religion unter veränderten Bedingungen - man könne „,nicht einfach sagen, dass mit der Aufklärung eine Entzauberung der Welt verbunden gewesen wäre, so als ob diese von einem Schleier des Religiösen nur befreit werden musste ${ }^{\text {“33 }}$. Gerade im säkularen Staat werde Religion nicht einfach bedeutungslos, sondern der Glaube unterliege einem bis heute andauernden Wandel.

Für Pädagogik als Praxis und Erziehungswissenschaft als deren wissenschaftliche Reflexion ergibt sich hieraus die Aufgabe und Schwierigkeit, den hier skizzierten Bedeutungswandel zu verstehen, mit zu vollziehen und in Konzepte von Bildung und Erziehung einfließen zu lassen. Dabei sind die Dimension der

$\begin{array}{ll}31 & \text { Vgl. Maaser 2007, S. } 92 \mathrm{f} . \\ 32 & \text { Vgl. Taylor 2009. } \\ 33 & \text { Ebd. }\end{array}$


Erfahrung auch im lebensweltlichen Alltag und die Schlussfolgerungen, die Menschen daraus ziehen, von Bedeutung.

\section{Die Dimension der Erfahrungen mit religiösen Kernüberzeugungen in multikulturell geprägten Regionen}

In multikulturell geprägten Ballungsräumen und Stadtgesellschaften (v.a.) machen Menschen im sozialen Nahraum Erfahrungen unterschiedlichster Art und Reichweite, die ihre jeweiligen Kernvorstellungen, mit denen sie sich stark identifizieren, berühren, beeinflussen oder gar in Frage stellen, z. B.:

- Die Erfahrung, dass die eigene religiöse (oder „nicht-religiöse“) Überzeugung nicht selbstverständlich von einer Mehrzahl der anderen geteilt wird. Diese Erfahrung setzt sich auf der Ebene der organisierten Formen fort, wenn z. B. Kirchen oder Gemeinden den Prozess von einer ursprünglich unbestrittenen lokalen Hegemonie zu einer Diaspora unter anderen anerkennen müssen.

- Die Erfahrung, dass durch die gesellschaftliche Ausdifferenzierung und die mit ihr einhergehende religiöse Pluralisierung die gewohnten kulturellen Muster religiöser Praxis innerhalb einer Religionsgemeinschaft stark differieren können (zum Beispiel die Gottesdienstgestaltung).

- Die Erfahrung, dass die in der Herkunftsgesellschaft festgefügten tradierten und allgemeingültigen dominanten religiösen ,Sitten und Gebräuche“ im Migrationsprozess allein durch die Feststellung in Frage gestellt werden können, dass man nun auch mit anderen religiös begründeten Selbstverständnissen unmittelbar benachbart ist.

- Die Erfahrung, dass reziprok auch Aufnahmegesellschaften religiös pluralisiert werden.

- Die Erfahrung, mit anderen Religionen den gesellschaftlichen Raum teilen zu müssen, kann bei den zugewanderten wie bei den ansässigen ethnisch-religiösen Gemeinschaften zu fundamentalistischen Orientierungen verleiten.

- Die Erfahrung, dass die Vermittlung, Begründung und Weitergabe spezifischer Wissensbestände um Glaubensschriften, Liturgien, und religiöse Praxen uneinheitlich und zuweilen ungeklärt erscheinen.

- Die Erfahrung - etwa bei konfessionell unterschiedlichen Ehepaaren, Lebenspartnerschaften oder Elternkonstellationen -, dass Erwartungen an eine „selbstverständliche Synchronisierbarkeit“ zwischen den religiösen Vorschriften und Konventionen enttäuscht werden. Wenn interkonfessionelle Amtshandlungen zwischen Familienmitgliedern unterschiedlicher Religionszugehörigkeit aufgrund der emotional dichten Beziehungen von diesen erwünscht werden, z. B. bei Anlässen wie Beerdigungen oder Hochzeiten, dies aber nur schwer oder gar nicht realisierbar ist.

- Die Erfahrung, dass es keine quasi-neutrale Beobachterperspektive auch solcher (obiger) Erfahrungen geben kann, sondern immer auch Beteiligung, 
denn: Auch unter den Vorzeichen einer strukturellen Trennung von kirchlicher und staatlicher Macht erfahren selbst Menschen mit ausgeprägt säkularen Positionen, dass eine demonstrative Verlagerung von allem Religiösen in den Bereich des Privaten spätestens dann obsolet wird, wenn religiöses Leben sich mit dem öffentlichen Leben durchdringt.

Für professionelle Pädagogen und Pädagoginnen, die ja von solchen Erfahrungsdimensionen nicht frei sind, bedeutet dies, reflexive Kompetenzen und die Fähigkeit auszubilden, jeweils auch die Perspektive der Anderen nachzuvollziehen. Für Eltern bedeutet es, dass die Art und Weise, wie sie jeweils diese Erfahrungen verarbeiten, in die Erziehung ihrer Kinder einfließt.

Für Kinder und Heranwachsende vermitteln sich derartige Erfahrungen der Erwachsenen mit einem anderen Bedeutungshorizont, denn für sie gilt auf andere Weise als für Erwachsene, dass die Dimensionen von Religiosität sich erst in einem Prozess der Aneignung und Entwicklung vollziehen. Dieser Aneingnungsprozess ist eingebettet in Erfahrungen der ganz persönlichen „Entdeckung“ von Transzendenz und Glaubensfragen, der Erfahrungen entsprechender Erziehungspraktiken und Erziehungsinhalte, der erfahrenen Sozialisation und religiöser Rituale im Umfeld der Familie. Im Rahmen späterer öffentlicher Erziehung und sekundärer Sozialisation kommen Erfahrungen im Kindergarten und der Schule, im Freundeskreis usw. hinzu. Kinder machen hier möglicherweise Erfahrungen, die auch ohne eine ,interreligiöse Erziehung“ in ganz unterschiedlicher Weise wirksam werden, z.B.:

- Die Erfahrungen in der Interaktion mit den eigenen Eltern und - später weiteren „signifikanten Anderen“ in Hinblick auf deren religiöse Authentizität. Steht die Glaubwürdigkeit dieser relevanten Bezugspersonen in Frage zumal in der Pubertät ein durchaus normaler Prozess der Identitätsfindung durch Abgrenzungen - kann auch leicht die religiöse bzw. säkulare Einforderung von Erwachsenen, das Weitergegebene nun auch zu beherzigen, in Mitleidenschaft gezogen werden.

- Die über die Eltern im primären Sozialisationsverlauf vermittelten religiösen Bezüge können abgelehnt, variiert oder puristisch als „wahre Religionsausübung" in der Arena intergenerativer Konflikte ausgetragen werden.

- Die Erfahrung über die offenen und verdeckten Redensarten, Deutungen und Urteile der Erwachsenen gegenüber rechten und unrechten Glaubensformen und Praxen Anderer. Studien zur Weitergabe von Vorurteilen ${ }^{34}$ die intergenerativ gelernt werden, geben hier Aufschluss. Vorurteile, ablehnende Haltungen oder Zustimmung werden auch affektiv, etwa durch körpersprachliche Signale der Eltern, die Kinder zuweilen sehr präzise registrieren können, erlernt und weitergegeben.

Vgl. Ahlheim 2009. 
- Die Erfahrung der Differenz und deren Stellenwert: Es gibt verschiedene Religionen, verschiedene Feiertage und Feste, verschiedene Gotteshäuser. Vor allem verschiedene häusliche und familiäre Umgangsformen, die sich als Sozialisationserfahrung manifestieren. (Zu Hause gibt es kein Tischgebet, aber bei der Freundin, wenn man dort zu Besuch ist.) Doch die Bedeutung dieser Verschiedenheiten, ihr Stellenwert scheint - anders zum Beispiel als bei einfach verschiedenen Farben, Tieren oder Spielen - für die Erwachsenen aus der Beobachtung der Kinder heraus schwerer zu wiegen als die der anderen Verschiedenheiten.

- Die Erfahrung der unterschiedlichen Deutung und Begründung im Zusammenhang mit religiösen Vorschriften. Im wechselseitigen Besuch kann erkannt und gelernt werden, dass es offenbar unterschiedliche Formen für denselben Zweck geben kann: Die einen ehren Gott, indem sie beim Gottesdienst den Kopf mit einer Kappe bedecken, den anderen ist es Pflicht, die Kappe auszuziehen, wenn Gott Ehre erwiesen werden soll. Solche Erfahrungen bergen das Potential, die jeweiligen kulturellen Vorschriften im religiösen Kontext zu relativieren im guten (Toleranz) wie im problematischen Sinne (Beliebigkeit). Erwachsene und Erzieher sind allemal gefordert, die jeweilige Praxis zu erläutern und zu plausibilisieren, ohne die jeweils andere Praxis einer anderen Religion zu diskreditieren. Dies setzt voraus, über das Eigene Bescheid zu wissen. Gerade im interreligiösen Vergleich kann sich solches Wissen profilieren und unter Umständen neu herausbilden.

- Die Erfahrung der Etikettierung: Du bist „katholisch“ oder eine „Jüdin“ oder „Moslem“. Wir sind „evangelisch“, was bist Du? Wohlgemerkt, dieses „Sein“ entsteht in einem Alter, in dem die zuvor angesprochenen legitimen Überzeugungsgewissheiten erst im Entstehen begriffen sind. Die Ontologisierung von religiösen Zugehörigkeiten ist in ähnlicher Weise problematisch wie eine kulturalistische Zuschreibung. Freilich ist es gerade eine Eigenart und ein berechtigtes Privileg der Zugehörigkeitsmaßstäbe zur Religionsgemeinschaft, sich diese nicht erst kognitiv - vernünftig - erschließen zu müssen, sondern etwa über die Taufe bei Christen oder die Beschneidung bei Juden - von der bereits bestehenden Gemeinschaft in Verbindung zu dem jeweiligen Gottesbezug schon als Säugling verbürgt zu erhalten.

- Die Erfahrung, dass die Identitätsmuster der Eltern oder eines Elternteils die Konversion des Kindes nahelegen oder erforderlich machen. In der Regel werden Konversionshandlungen vorgenommen, wenn sie mit der Perspektive verknüpft werden, die angestrebte Zugehörigkeit $\mathrm{zu}$ einer religiösen Gemeinschaft einlösen zu können. Die formale Konversion bedeutet in vielen Fällen nicht, dass die soziale Akzeptanz in der neuen Gruppe unmittelbar nach dem Konversionsakt gewährleistet ist. Entsprechende Differenzerfahrungen haben besonders für Kinder irritierende Konsequenzen, da die religiöse Neuorientierung oft auch veränderte Interaktionsprozesse nach sich zieht. Konvertiten unterliegen häufig einem selbstgesetzten erhöhten Anpassungsdruck 
und wähnen sich unter verschärfter Beobachtung durch die aufnehmende Gemeinde, was sich auf die Kinder überträgt.

- Die Erfahrung der unzulässigen Vermischung und Verzahnung von unakzeptablen Erziehungspraktiken, z.B. Kindeswohlmissachtung durch strafende, misshandelnde oder sonstige, die Integrität des Kindes beeinträchtigende Handlungen, mit religiösen Rechtfertigungen. ${ }^{35}$

- Die Erfahrung für Jugendliche, mittels religiös motiviertem bzw. legitimiertem Rigorismus mögliche Probleme bei der Adoleszenz bewältigen zu können. In diesen Fällen spielen eher fundamentalistische Verlockungen denn religiöse Substanz eine Rolle. Beispiele sind die Faszination durch Sekten oder militarisierte politisierte Religion.

Kinder machen Erfahrungen religiöser Differenz v. a. vermittelt über die Haltungen und Wertungen ihrer erwachsenen Bezugspersonen. Wenn sie aus Sicht ihrer Eltern oder Erziehungsberechtigten, ihrer Gemeinschaften oder kirchlichen Mitgliedschaft jeweils als Zugehörige einer bestimmten Religion behandelt und bezeichnet werden, verbürgt dies zunächst eine positive Bestimmung im Sinne der jeweiligen Religion: Zum Beispiel eine Einbettung in die sozialen Bezüge und Praxen der jeweiligen Religionsgemeinschaft und eine Rhythmisierung menschlicher Zeitabläufe anhand von Festen und Feiertagen. Es ergeben sich aber auch Risiken der sozialen Etikettierung. Entsprechende Erfahrungen des Ausgeschlossen-Werdens betreffen in gleicher Weise Kinder von nicht- oder antireligiösen Eltern oder Erziehern. (Warum kriegen die Katholiken Plätzchen in der Kirche? Warum feiern wir kein Weihnachten? Warum haben die Muslime heute frei? Warum bekomme ich nichts vom Nikolaus? usw.)

Für Jugendliche kann Religion in puristischer Auslegung ein Bewältigungsmuster in den Krisen der Adoleszenz darstellen.

\section{Pädagogische Implikationen zwischen Zumutung, religiösem Gehorsam und Mündigkeit}

Wenn Erwachsene die ihnen anvertrauten Kinder interreligiös erziehen wollen, müssen sie sich zunächst verständigen, entscheiden und darüber klar werden, welche Art religiöser Erziehung sie anstreben. Sie sollten dabei vermeiden, eigene ungelöste Fragen im Zusammenhang eigener noch unbewältigter Erfahrungen im religiösen und nicht-religiösen Miteinander (s.o.) als „Hypothek“ an die Kinder und Heranwachsenden zu delegieren, wohl aber sollten sie authentisch bleiben und den Nachkommen sowohl offene Fragen wie eigene Gewissheiten nicht vorenthalten. Religiöse Erziehung - und damit auch jede interreligiöse Erziehung - ist von einer allein religionskundlichen Wissensvermittlung vor allem

Vgl. Brumlik 2010, S. 185. 
dadurch unterschieden, dass sie im Modus authentischer Repräsentationen erfolgt. Von professionellen Pädagogen und Pädagoginnen ist dabei eine reflexive Authentizität zu entwickeln, die ein Bekenntnis zur eigenen religiösen Verortung nicht scheut, vor allem, wenn Kinder danach fragen; einschließlich solcher Positionen, die sich als Zweifelnde oder Suchende ausgeben. „Reflexive Authentizität“" schließt eine Förderung „,religiöser Dialog- und Urteilsfähigkeit“ etwa im schulischen Religionsunterricht ein, wie sie aus katholischer Perspektive etwa Langenhorst zur Diskussion stellt: Neben der Fähigkeit, religiöse Phänomene überhaupt wahrnehmen zu können, begrifflich fassen, verstehen und sprachlich darstellen zu können, setzt „religiöse Dialog- und Urteilsfähigkeit“ eine religiöse Deutungskompetenz voraus, also die Fähigkeit, „in religiösen Fragen rational begründet urteilen zu können“. Religiöse Urteilsfähigkeit gilt dabei als „unabdingbare Voraussetzung zu religiöser Mündigkeit und Selbstbestimmung “36.

Gleichzeitig ist eine Erziehung zur Toleranz in ,interreligiöser“ Perspektive auf Gebote zur Toleranz innerhalb der religiösen Überlieferungen und Vorschriften verwiesen. Sofern Religionen, ihre (Vor-)Schriften und Interpretationen in ihrer Substanz keine Optionen zur Tolerierung anderer aufweisen, sind dem interreligiösen Dialog Grenzen eingeschrieben. Der Zielhorizont einer interreligiösen Auseinandersetzung liegt also noch nicht fertig vor und es sieht nicht danach aus, dass dies ohne Weiteres in absehbarer Zukunft gelingt, aber es lassen sich Verhältnisbestimmungen und unterscheidbare Richtungen rekonstruieren, die auch die bisher in der Geschichte ,interreligiöser“ Beziehungen stattgefundenen Prozesse und Erfahrungen aufzunehmen hätte einschließlich ihres Scheiterns in zerstörerischen Kämpfen.

Religiöse Kernauffassungen sind von ihrem Selbstverständnis her notwendigerweise von ihrem ausschließlichen Wahrheitskonzept überzeugt und stellen somit die Sichtweisen anderer Religionen in Frage. Interreligiöse Annäherungen und Auseinandersetzungen sind daher anstrengend und nicht selten eine $\mathrm{Zu}$ mutung für alle Beteiligten. Zugleich ist der interreligiöse Dialog dafür prädestiniert, Interaktionsmuster unter Individuen und Gruppen einzuüben, die die Grundlage einer Koexistenz in einer liberalen demokratischen Gesellschaft darstellen: das Ertragen von differenten Überzeugungen, die nicht notwendigerweise zum Abbruch der Beziehung führen muss; die Entdeckung, dass Individuen unterschiedliche Teilidentitäten repräsentieren, von denen die religiöse nur eine unter vielen beschreibt; die Einsicht, dass auch wenn die eigenen Gewissheiten von anderen nicht geteilt werden, bestimmte lebensweltlich relevante und sich überschneidende Perspektiven den gemeinsamen Alltag strukturieren.

Für die pädagogische Praxis soll vor diesem Hintergrund auf folgende Aspekte aufmerksam gemacht werden:

- Wenn von ,interreligiöser Erziehung“ als Herausforderung der Pädagogik die

Rede ist, muss bedacht werden, dass die Herausforderungen in der Sache selbst

36 Langenhorst 2014, S. 188. 
(interreligiöse Beziehungen) stärker wiegen als die spezifisch pädagogischen Aufgaben. Aus einer pädagogischen Perspektive ist dem Kindeswohl daher immer ein Primat einzuräumen. Brumlik weist auf die Alternative hin, nach der sich „die Frage nach Grenzen und Möglichkeiten religiöser Erziehung [...] entweder aus der Perspektive religiöser Überzeugungen bzw. theologischer Wahrheiten oder aus der Perspektive des Kindes stellen“"37 lässt, wobei die Forschung für Kinder zwischen dem 3. und 7. Lebensjahr in einem vor allem durch die Eltern geprägten sozialisatorischen Umfeld einen ,intuitiv-projektiven Glauben“ beobachtet hat. ${ }^{38}$ Die kindliche Religiosität beginnt sich anhand symbolischer Erzählungen und Rituale zu formen und ist gleichsam gefährdet, wenn anhand des Bildes eines übermächtigen, alles erkennenden, strafenden Gottes übersteigerte moralische Tabus und Schuldgefühle entstehen und sich in einem rigiden Über-Ich des Kindes manifestieren.

- Ziele und Konzepte einer „interreligiösen Erziehung“ können an Konzepte interkultureller Pädagogik ${ }^{39}$ und eine migrationspädgogische Erziehung zur Toleranz ${ }^{40}$ zwar angeschlossen werden, gehen darin aber nicht auf. Denn der Zusammenhang von religiöser Praxis als zugleich kultureller Praxis bedeutet noch nicht, dass Religionsausübungen allein eine Frage kultureller Orientierung, Konvention oder Neuschöpfung wäre. Kulturelle Praxis als spezifische menschliche Eigenart beinhaltet Symbolisierungen, Ausdruckshandeln, Formfindung und Formgebungen, und nach einer kulturtheoretischen Figur des Philosophen Ernst Cassirer stehen das menschliche Selbst, seine Werke und deren Wirkungen in einem spannungsreichen Wechselverhältnis, wobei „Kultur“ immer als dynamischer Prozess eben jener Kulturtätigkeit gesehen wird. Religionen gehören im Bild dieser Kulturtheorie zum Netz symbolischer Formen, die sich der Mensch - wie die Sprache - als zweite Realität erschaffen hat. ${ }^{41}$ Die für die interkulturelle Perspektive so zentrale Kategorie der Verhandelbarkeit kultureller Ansprüche über Wege der Verständigung und eines annähernden und anerkennenden Verstehens kultureller Orientierungen geht im (inter)religiösen Kontext aber nur soweit auf, als religiöse Urteile sozial wirksam werden und einer kognitiven Entwicklung folgen können, in der der religiöse Mensch von einer „Orientierung an einem Letztgültigen, das direkt und unmittelbar in die Welt eingreifen kann und vom Menschen kaum beeinflussbar ist ${ }^{\text {“42 }}$, zu einer Stufe religiöser Gewissensbildung gelangt, in der das sich als vollständig autonom und verantwortlich verstehende und akzeptie-

\footnotetext{
37 Brumlik 2010, S. 184.

38 Vgl. Fowler 1991.

39 Vgl. Auernheimer 2007.

40 Vgl. Diehm 2000.

41 Vgl. Eppenstein 2003, S. 85-87.

42 Brumlik 2010, S. 188.
} 
rende Individuum die Sphären des Göttlichen und Menschlichen klar zu trennen beginnt.

- Aus ethischer Perspektive konkurrieren mindestens zwei Auffassungen um die Möglichkeit der Toleranz zwischen verschiedenen Religionen. Die eine erkennt den „einen Gott“ jeweils auch als Gott der anderen mit ihren je unterschiedlichen Formen menschlicher Religionsauffassung und Religionsausübung, die andere erkennt grundlegende Differenzen und Unterschiedlichkeiten, die zwar unaufhebbar, aber doch wechselseitig tolerierbar erscheinen. Während die erste Auffassung Gefahr läuft, der Toleranzaufforderung durch Relativierungen und eine inhaltlich entleerte religiöse Identität nachzukommen, erfordert die zweite, die die Differenzen nicht verblassen lässt, „der unvermeidlichen religiösen Kontextuierung der beteiligten Menschen Rechnung “43 zu tragen. Den religiös Beteiligten wird nicht sofort eine Beobachterperspektive angetragen, ,sondern die spezifischen Praktiken und inhaltlichen Dimensionen in ihrem gewissheitsstiftenden und Orientierung gebenden Charakter ernst" ${ }^{\text {44 }}$ genommen.

Mit Blick auf „interreligiöse Aufgaben“ sollten Pädagogen daher nicht nur die Differenz und mögliche Übereinstimmungen in und zwischen „den Religionen“ fokussieren, sondern deren Bedingungen zu einer Entwicklung autonomer Gewissensbildung beachten: Brumlik benennt einige Voraussetzungen, die im Kontext religiöser Erziehung Beachtung finden sollten. So sollte die Vermittlung religiöser Orientierungen und Überzeugungen frei von angsteinflößenden Ankündigungen oder strafenden Gottesbildern bleiben. Des Weiteren sollten religiöse Wissensbestände problemorientiert und gegebenenfalls als moralische Dilemmata eingeführt werden. Eine authentische und an den jeweiligen Lebenserfahrungen angelehnte religiöse Erziehung befördert die Entwicklung der autonomen Gewissensbildung unter Einbindung religiöser Traditionen und einer religiös begründeten Moral. ${ }^{45}$

Es heißt, Eltern seien das Schicksal ihrer Kinder. Eine Alltagsweisheit erinnert daran, dass man sich „Freunde im Leben aussuchen kann, nicht aber die eigenen Eltern“. So gesehen werden Kinder und Eltern im Familienzusammenhang einander zugemutet, und im Verlauf des Heranwachsens greifen die Zumutungen auf die Akteure öffentlicher Erziehung über, auch wenn ihnen gegenüber andere Möglichkeiten der Distanzierung respektive Identifikation möglich werden und eine andere Struktur von Distanz-Nähe-Bestimmungen Platz greift. Pädagogisch gewendet oszillieren Erziehungsprozesse zwischen Zumutung und Zutrauen, und was da jeweils zugemutet bzw. zugetraut wird, changiert zwischen den jeweiligen Erziehungszielen, also den Absichten und Wünschen der Erzieher die Educandes

43 Maaser 2007, S. 101.

44 Ebd.

45 Vgl. Brumlik 2010, S. 189-191. 
betreffend, und den Eigenarten der Erziehenden selbst, die sich die Heranwachsenden über mehr oder weniger gelingende Formen der Bindung in Interaktionen erschließen. Aus der Perspektive von Kindern können phasenweise die religiösen Kernvorstellungen ihrer Bezugspersonen „heilig“ oder „unheilig“" sein, weil sie eben von dieser spezifischen Person verkörpert und nicht nur vertreten werden: dem nahen oder fernen Elternteil, der heimlich verehrten oder offen verlachten Lehrerin, dem geachteten oder auch gefürchteten oder fremdartigen Religionsvertreter usw.

Die Tatsache dieser „strukturellen Zumutung“ ist allein deshalb unhintergehbar, weil Erziehungsprozesse stets in einem asymmetrischen Machtgefüge zwischen Erzieher und Erzogenem stattfinden. Begrifflich zu unterscheiden sind dabei Erziehungsverständnisse, Erziehungshandlungen, Erziehungsstile und Erziehungsmittel, denen es stets um eine zu legitimierende Form der Intervention, also um Eingriffe geht. Während „Erziehung“ als Prozess des „Sozial-Machens“ normativ an positiv zu begründeten Zielen ausgerichtet ist, geht es in Bildungsprozessen um günstige Arrangements, in denen der Selbstbildungsprozess von Individuen - auch Kindern (!) - angeregt und gefördert wird. Der „Kindergarten“, die Kita, Schulen usw. sind Bildungsinstitutionen und Bildungsräume, aber gleichzeitig auch Sozialisationsorte, wobei „Sozialisation“ in Abgrenzung zur „Erziehung" eher beschreibend den Prozess von Individuation und Vergesellschaftung, also des „Sozial-Werdens" meint.

Macht man sich diese Tatsache der Unumgehbarkeit wechselseitiger Zumutungen unter Voraussetzungen ungleicher „Augenhöhe“ bewusst, wird begründbar, dass Erziehungshandlungen später vor den dann einmal Erzogenen rechtfertigbar sein sollten: Dies geschieht gemeinhin in Bezug auf die Person, ihre Haltungen und Handlungen und in Bezug auf die je vertretene "Sache“. Von mündigen Erwachsenen, die die zu erziehenden Kinder ja einmal werden sollen, kann erwartet werden, dass sie gelernt haben zwischen Achtung der Person und Bewertung einer sachbezogenen Position zu unterscheiden. So mag z. B. ein Vater in seinen politischen Auffassungen durchgängig überzeugen, aber als Vater war er - aus der Perspektive seines Kindes - ein menschlicher Versager; oder umgekehrt: politisch lag er vollkommen daneben, aber das beeinträchtigt nicht die Achtung seiner Person. Sofern hier Differenzen oder Übereinkünfte in Fragen der Weltanschauung, der politischen Positionierung, der erfahrenen Erziehungsformen, der Persönlichkeitsmerkmale oder der je spezifischen Beziehungsqualitäten usw. angesprochen sind, scheint die Sache relativ einfach, weil hier die jeweiligen individuellen Dispositionen, Urteile und - mehr oder weniger freien - Entscheidungsprozesse normativer und sachlicher Art einfließen.

Wie aber steht es mit dem Verhältnis von Person und „Sache“ im Zusammenhang mit dem Religiösen, dass ja mehr zu sein beansprucht, als eine subjektiv zu erlangende oder auch zu verwerfende Positionierung? Im interreligiösen Vergleich fallen die Antworten unterschiedlich aus, je nachdem, ob „Gott“ etwa als letztlich alle und alles beherrschende Zentralgewalt gesehen oder geglaubt 
wird, oder als dialogisch zu erschließendes Gegenüber, ob eher als „göttliches Prinzip“ oder als „,ansprechbarer Vater im Himmel“ usf.

Oft fordern religiöse Grundsätze „Gehorsam“ und kollidieren dabei mit Prinzipien einer Erziehung zur Mündigkeit, die sich seit Beginn der Neuzeit und Aufklärung als zentrales Paradigma - zumal in den westlichen Gesellschaften herausgebildet hat. Unter dieser Perspektive wird Erziehung ,vom Kinde aus“, also aus der Perspektive des Kindes bedeutsam und die oft überraschenden „naiven“ Auslegungen, Fragen und Feststellungen von Kindern zu ihrer je eigenen, sich entwickelnden Religiosität, können sie durchaus als ihrerseits „,(inter)religiös Lehrende“ für Erwachsene auftreten lassen. Dies setzt jedoch schon eine bestimmte Auffassung von zulässiger religiöser Praxis seitens der Erwachsenen voraus. So ist etwa entscheidend, ob die jeweiligen Textüberlieferungen für eine Interpretation durch Menschen verfügbar sind oder nicht, ob ein dialogisches und erörterndes Verständnis nicht nur zwischen Kindern und Erwachsenen, sondern auch im Spannungsverhältnis von Vernunft und Offenbarung, von Mensch und Gott, von Gehorsam als Vorgabe und Gehorsam im Sinn eines lebensdienlichen Gebotes zugrunde gelegt wird.

Die strukturelle Zumutung durch die jeweilige religiöse oder auch areligiöse Position von Eltern gegenüber den zu erziehenden Kindern wird im Feld des Religiösen gerade dadurch unhintergehbar, dass religiöse Grundsätze selten verhandelbar daherkommen; allein dadurch erhalten sie Gewicht und werden zu einer Art Währung, die aus dem Zugemuteten gleichzeitig „Zuspruch“ in einem sehr grundsätzlichen und tiefen Sinne werden lässt. Die so entstehenden scheinbaren Paradoxien können einen Sinn erhalten: Wer „gottesfürchtig“ ist, braucht vielleicht weniger Furcht vor den Wechselfällen des Lebens haben, oder: Das Verhältnis von Gehorsam gegenüber Gott und/oder den Menschen und die vielfältigen Erörterungen hierzu geben Aufschluss darüber, dass hier „Gehorsam" gleichsam Freiheitserfahrungen und Freiheitsoptionen verbürgt.

Man kann postulieren, dass Kinder und Heranwachsende unter den uneindeutigen Bedingungen einer ,flüchtigen Moderne“ “46 , die die religiöse Vielfalt im demokratischen Rechtstaat erst unter den Bedingungen einer Gewaltenteilung zwischen Staat und den verfassten Religionsgemeinschaften ermöglicht, geradezu ein Recht auf derlei Zumutungen haben, an denen sie sich orientieren, reiben und wachsen können. Freilich gilt auch, dass mit dem heutigen Wissen um ein gedeihliches Aufwachsen von Kindern, von Entwicklungsprozessen, Bewältigungsaufgaben im Kindes- und Jugendalter, widrigen und günstigen Sozialisationsbedingungen und psychosozialen Prozessen ein Kurzschluss zwischen religiös oder kirchlich gebotenem „Gehorsam“ auf der einen- und einem auf (Kadaver)gehorsam setzenden, autoritären Erziehungsstil auf der anderen Seite in den meisten Fällen nicht nur die Rechte des Kindes verfehlt, sondern auch den Kern dessen, was als religiös gebotenes Substrat auf dem Wege der Erziehung weiter-

Vgl. Baumann 2003. 
gegeben werden soll. Eine strafpädagogische Praxis ist grundsätzlich nicht mit Verweis auf einen - auch möglicherweise strafenden - Gott legitimierbar.

\section{Konklusionen}

Durch Modernisierungsprozesse, die eng mit Migrationen und Erfahrungen kultureller Pluralisierung verbunden sind, entstehen interreligiöse Berührungsund Reibungspunkte sowohl im sozialen Nah-Raum, als auch in transnationalen sozialen Netzen. Dies wirft Fragen nach interkulturell kompetenten und interreligiös informierten und aufgeschlossenen Bildungskonzepten auf. Von professionellen Pädagogen und Pädagoginnen erfordert dies eine Klärung von Haltungen und Kompetenzen, Reflexivität und eine Erweiterung ihrer Wissensbestände. Dabei ist zu berücksichtigen, dass religiöse Kernüberzeugungen im Vergleich zu kulturellen Orientierungen oder politischen Überzeugungen kaum verhandelbar sind, mithin Klärungsbedarf besteht, was zwischen den Religionen $\mathrm{zu}$ lernen ist und welche Grenzen dabei bereits strukturell und von der Sache her eingeschrieben sind. Deskriptionen von Dialogformen, die unter dem Signum „interreligiös“ zu beobachten sind, sind jedoch empirisch möglich.

„Religiosität“ ist nicht allein immanent in Hinblick auf die jeweiligen Kernüberzeugungen und Lehren von Bedeutung, sondern immer auch sozial relevant. Sie findet ihre Form(en) - z. B. in den jeweiligen Gemeinden - in sozialen Interaktionen, Kommunikationen und Konventionen auch dann, wenn sie - wie vor allem in den westlichen Gesellschaften - vorrangig individuell angeeignet wird.

Interreligiöse Erziehung erfordert von professionellen Pädagogen zunächst pädagogische, entwicklungspsychologische oder auch sozialisationstheoretische Kenntnisse zur Wahrung kindlicher Integrität und des Kindeswohls und sie erfordert interkulturelle Kompetenzen. Darüber hinaus verlangt sie nach einer bewusst zu treffenden Entscheidung (meist der Eltern), ob, wo, und wie die Kinder religiös zu erziehen seien. Auf dieser Grundlage treten die Professionellen nicht allein als Vermittler, evtl. religionskundige kenntnisreiche Experten oder als quasi-neutrale Beobachter auf, sondern agieren auch immer als Zeugen ihrer jeweiligen religiösen Wahrheit im Sinne einer reflexiven Authentizität. Erst wer gelernt hat, die Sphären des göttlichen von denen des menschlichen im Sinne menschlicher Verantwortungsübernahme auch zu unterscheiden, wird gegenüber Heranwachsenden nicht als Wahrheitsbesitzer auftreten und entgeht damit der Gefahr, gewissermaßen selbst Gott zu spielen oder ihn ,als dessen Werkzeug“ für beliebige Interessen zu instrumentalisieren.

Grundlegende Erziehungsziele zur Mündigkeit, die ja Kategorien der Freiheit, Vernunft und Urteilsbildung einschließen, kollidieren nicht grundsätzlich mit religiöser Erziehung. Entsprechende Sozial- und Persönlichkeitskompetenzen können sich in interreligiösen Beziehungen herausbilden. Denn eine Norm des „Gehorsams“ hat pädagogisch nicht zwingend einen autoritären Erziehungsstil 
zur Folge, sondern kann - im Wortsinn - als „Befolgen“ des „Gehörten“ die Dimension der Konsequenzen ernst nehmen, die aus einem Bekenntnis zu einer bestimmten Religion und der daraus gewonnenen gruppenbezogenen sozialen und religiösen Praxis resultieren. Für die pädagogische Praxis gilt die Einsicht in die Bedeutung der Dimensionen des tradierten Wissens wie des aktuellen Erlebens, die mit der religiösen Praxis zum Ausdruck kommen. Wenn diese Wissens- , Traditions- und Erlebensbereiche im Kindesalter gleichzeitig erworben, erfahren und mit anderen - interreligiös - ausgetauscht, verglichen, zuweilen auch geteilt werden, tragen Erwachsene die Verantwortung, dass dies als Aneignung von Welt angstfrei gelingt, ohne die Kinder für eigene Zwecke zu instrumentalisieren und ihre Integrität zu verletzen.

In multireligiösen Gesellschaften entstehen entsprechend neue Bildungsaufgaben, die religiös fundierte Menschen wie Nichtreligiöse gleichermaßen adressieren. Bei der Klärung gemeinsamer Normen gilt pädagogisch ein religiöses Überwältigungsverbot, ohne dass dabei „Religion auf eine bloße Privatangelegenheit reduziert ${ }^{\text {“47 }}$ werden braucht.

Für Bildungseinrichtungen und Bildungskonzepte von Religionsgemeinschaften stellen sich bedingt durch Migration und Pluralisierung folgende Klärungsaufgaben:

Prozesse der inneren Pluralisierung und Differenzierung, die historisch immer wieder zu Abspaltungen geführt haben, werden in Migrationskontexten innerhalb einer Religionsgemeinschaft besonders auffällig, wenn sie mit unterschiedlichen kulturellen Praktiken und Konventionen einhergehen, die nicht unbedingt aus der religiösen Praxis selbst, sondern als Amalgam aus internalisierter Kultur und Gruppenzugehörigkeit entstehen. Hier müssen für die Angehörigen der Religionsgemeinschaften geeignete Umgangsformen gefunden und erlernt werden, Vielfalt und Vielstimmigkeit innerhalb der eigenen Gruppe auch dann anzuerkennen, wenn diese als Zumutung oder fremd empfunden wird.

Gleichzeitig müssen eindeutige Abgrenzungen dann vorgenommen werden, wo nicht differierende kulturelle Traditionen im religiösen Leben oder Auslegungsfragen religiöser Texte maßgeblich sind, sondern eine Instrumentalisierung von Religion für fundamentalistische Projekte zu befürchten ist.

Bildungsanstrengungen von verantwortlichen Akteuren aus den Religionsgemeinschaften haben nicht mehr allein das Ziel zu verfolgen, die jeweiligen religiösen Wissensbestände zu tradieren, die ihnen innewohnenden Gesetze und Praktiken, Vollzüge und Bedeutungen von religiösen Festen und Feiertagen usw. zu vermitteln und weiter zu geben. Sie müssen darüber hinaus ihr Verhältnis im säkularen Rechtsstaat und gegenüber anderen Religionsgemeinschaften klären oder geklärt haben und dabei einen Lernprozess lebendig erhalten, der als Bildungsziel tragfähige Formen wechselseitiger Anerkennung und Respekt verfolgt.

47 Schweitzer 2016, S. 180. 


\section{Literaturverzeichnis}

Ahlheim, Klaus (Hg.): Die Gewalt des Vorurteils. Schwalbach am Taunus 2009.

Amos, Karin: „Interreligiöse und interkulturelle Kompetenz in der Ausbildung für den Elementarbereich. Response aus erziehungswissenschaftlicher Sicht“, in: Biesinger, Albert / Schweitzer, Friedrich (Hg.): Kulturell und religiös sensibel? Münster / New York 2015, S. 163-172.

Auernheimer, Georg: Einführung in die Interkultuelle Pädagogik. Darmstadt 2007.

Bade, Klaus J.: Kritik und Gewalt. Schwalbacht am Taunus 2013.

Bahners, Patrick: Die Panikmacher. Die deutsche Angst vor dem Islam. München 2011. Baumann, Zygmunt: Flüchtige Moderne. Frankfurt am Main 2003.

Bohmeyer, Axel: „Soziale Arbeit und Religion - sozialwissenschaftliche und anthropologische Spurensuchen in postsäkularer Gesellschaft“, in: neue praxis (5) 2009, S. 439-450.

Bielefeld, Heiner: „Religiöser Pluralismus im säkularen Rechtsstaat“, in: Nagel, Helga / Mechthild Jansen (Hg.): Religion und Migration. Amt für multikulturelle Angelegenheiten der Stadt Frankfurt am Main und Hessische Landeszentrale für politische Bildung. Frankfurt am Main 2007, S. 21-28.

Brumlik, Micha: „Sollen wir unser Kind religiös erziehen, und wenn ja -wie?“, in: Andresen, Sabine / Brumlik, Micha / Koch, Claus (Hg.): Das Elternbuch. Weinheim / Basel 2010, S. 184-195.

Diehm, Isabell: „Erziehung und Toleranz. Handlungstheoretische Implikationen Interkultureller Pädagogik“, in: ZfPäd (46/2) 2000.

Eppenstein, Thomas: „Interkulturelle Kompetenz - Zumutung oder Zauberformel“, in: Zacharaki, Ioanna / Eppenstein, Thomas / Krummacher, Michael (Hg.): Praxishandbuch interkulturelle Kompetenz. Schwalbach am Taunus 2007/2013, S. 35-66.

Eppenstein, Thomas: Einfalt der Vielfalt? Interkulturelle pädagogische Kompetenz in der Migrationsgesellschaft. Frankfurt am Main 2003.

Eppenstein, Thomas / Kiesel, Doron: Soziale Arbeit interkulturell. Stuttgart 2008.

Fowler, James: Stufen des Glaubens. Die Psychologie der menschlichen Entwicklung und die Suche nach Sinn. Gütersloh 1991.

Habermas, Jürgen: Glauben und Wissen. Frankfurt am Main 2001.

Jammer, Max: Einstein und die Religion. Konstanz 1995.

Joas, Hans: Die Sakralität der Person. Eine neue Genealogie der Menschenrechte. Berlin 2011.

Joffe, Josef: Mach dich nicht so klein, du bist nicht so gross! Der jüdische Humor als Weisheit, Witz und Waffe. München 2015.

Kaufmann, Franz-Xaver: „Religion zwischen Tradition, Selbsterfahrung und Dauerreflexion“, in: Schmidt, Thomas M. / Parker, Michael G. (Hg.): Religion in der pluralistischen Öffentlichkeit. Würzburg 2008, S. 21-39.

Knoblauch, Hubert: „Einleitung: Soziologie der Spiritualität“, in: Zeitschrift für Religionswissenschaft (13) 2005, S. 123-131.

Langenhorst, Georg: Kinder brauchen Religion. Orientierung für Erziehung und Bildung. Freiburg im Breisgau / Basel / Wien 2014.

Luckmann, Thomas: Die unsichtbare Religion. Frankfurt am Main 1991. 
Maaser, Wolfgang: „Religionen und Toleranz - Dimensionen eines interkulturellen Problemfeldes“, in: Zacharaki, Ioanna / Eppenstein, Thomas / Krummacher, Michael (Hg.): Praxishandbuch Interkulturelle Kompetenz. Vermitteln, Vertiefen, Umsetzen. Schwalbach am Taunus 2007, S. 91-106.

Mecheril, Paul / Castro Varela, Maria do Mar / Dirim, Inci / Kalpaka, Annita / Melter, Claus: Migrationspädagogik. Weinheim / Basel 2010.

Mollenhauer, Klaus: Vergessene Zusammenhänge. München 1983.

Nagel, Helga / Jansen, Mechthild (Hg.): Religion und Migration. Amt für multikulturelle Angelegenheiten der Stadt Frankfurt am Main und Hessische Landeszentrale für politische Bildung. Frankfurt am Main 2007.

Oser, Fritz / Gmünder, Paul: Der Mensch - Stufen seiner religiösen Entwicklung: ein strukturgenetischer Ansatz. Gütersloh 1988.

Pries, Ludger: „Verschiedene Formen der Migration - verschiedene Wege der Integration“, in: Neue Praxis Sonderheft (8) 2006, S. 19-27.

Pries, Ludger: „The Disruption of Social and Geographic Space“, in: International Sociology (16/1) 2001, S. 51-70.

Rentsch, Thomas: „Negativität und Rationalität. Gibt es aus philosophischer Sicht irreduzible Wahrheitsansprüche religiöser Vernunft?“, in: Praxis Sonderheft (8) 2006, S. 161-178.

Riesebrodt, Martin: Die Rückkehr der Religionen. Fundamentalismus und der „Kampf der Kulturen“. München 2000.

Schambeck, Mirjam: Interreligiöse Kompetenz. Göttingen 2013.

Schmidt, Thomas M. / Parker, Michael G. (Hg.): Religion in der pluralistischen Öffentlichkeit. Würzburg 2008.

Schütz, Alfred / Luckmann, Thomas: Strukturen der Lebenswelt. Konstanz 2003.

Schweitzer, Friedrich: Das Bildungserbe der Reformation. Bleibender Gehalt, Herausforderungen, Zukunftsperspektiven. Gütersloh / München 2016.

Taylor, Charles: Ein säkulares Zeitalter. Frankfurt am Main 2009.

Weber, Max: Die protestantische Ethik und der Geist des Kapitalismus. München 1920/ 2004. 